PROCEEDINGS OF THE

AMERICAN MATHEMATICAL SOCIETY

Volume 131, Number 7, Pages 2217-2221

S 0002-9939(03)06863-1

Article electronically published on February 20, 2003

\title{
VECTOR BUNDLES WITH INFINITELY MANY SOULS
}

\author{
IGOR BELEGRADEK \\ (Communicated by Wolfgang Ziller)
}

\begin{abstract}
We construct the first examples of manifolds, the simplest one being $S^{3} \times S^{4} \times \mathbb{R}^{5}$, which admit infinitely many complete nonnegatively curved metrics with pairwise nonhomeomorphic souls.
\end{abstract}

According to the soul theorem of J. Cheeger and D. Gromoll [CG72], a complete open manifold of nonnegative sectional curvature is diffeomorphic to the total space of the normal bundle of a compact totally geodesic submanifold, called a soul. The soul is not unique but any two souls are mapped to each other by an ambient diffeomorphism inducing an isometry on the souls Sha79. In this note we show that the homeomorphism type of the soul generally depends on the metric; namely the following is true.

Theorem 1. There exist infinitely many complete Riemannian metrics on $S^{3} \times$ $S^{4} \times \mathbb{R}^{5}$ with sec $\geq 0$ and pairwise nonhomeomorphic souls.

The proof applies some classical techniques of geometric topology to recent examples of nonnegatively curved manifolds due to K. Grove and W. Ziller GZ00. As we explain below, it is much easier to produce a manifold with finitely many nonnegatively curved metrics having nonhomeomorphic souls, however the full power of [GZ00] is needed to get infinitely many such metrics.

Grove and Ziller [GZ00] showed that any principal $S^{3} \times S^{3}$-bundle over $S^{4}$ admits an $S^{3} \times S^{3}$-invariant metric with sec $\geq 0$. By O'Neill's formula, all associated bundles admit metrics with sec $\geq 0$ which gives rise to a rich class of examples, including all sphere bundles over $S^{4}$ with structure group $S O(4)$. Note that the souls in Theorem 1 1 are the total spaces of $S^{3}$-bundles over $S^{4}$ with structure group $S O(3)$. Theorem 1 is a particular case of the following.

Theorem 2. Let $\xi$ be a rank $n$ vector bundle over $S^{4}$ with structure group $S O(3)$, let $q: S \rightarrow S^{4}$ be a smooth $S^{m-1}$-bundle with structure group $S O(3)$, and let $\eta$ be the q-pullback of $\xi$. If $m=4, n>4$, or if $m>4, n>m+3$, then the total space of $\eta$ admits infinitely many complete Riemannian metrics with $\mathrm{sec} \geq 0$ and pairwise nonhomeomorphic souls.

The main topological tool used in this paper is a result of L. Siebenmann [Sie69] that generalizes the famous Masur's theorem: any tangential homotopy equivalence of closed smooth $n$-manifolds is homotopic to a diffeomorphism after taking the

Received by the editors June 17, 2001 and, in revised form, September 25, 2001.

2000 Mathematics Subject Classification. Primary 53C20.

Key words and phrases. Nonnegative curvature, soul. 
product with the identity map of $\mathbb{R}^{n+1}$. Exotic 7 -spheres are stably parallelizable, so they all become diffeomorphic after taking the product with $\mathbb{R}^{8}$, and in fact it suffices to take product with $\mathbb{R}^{5}$. A homotopy 7-sphere is called a Milnor sphere if it is diffeomorphic to the total space of an $S^{3}$-bundle over $S^{4}$; it is known that 10 out of 14 homotopy 7-spheres are Milnor. According to [GZ00, any Milnor sphere carries a metric with $\mathrm{sec} \geq 0$, which leads to the following.

Proposition 3. For every Milnor sphere $\Sigma$, the manifold $S^{7} \times \mathbb{R}^{5}$ has a complete Riemannian metric of $\mathrm{sec} \geq 0$ with soul diffeomorphic to $\Sigma$.

Gromoll and Tapp recently classified [GT01] all nonnegatively curved metrics on $S^{2} \times \mathbb{R}^{2}$, and asked for a similar classification on $S^{n} \times \mathbb{R}^{k}$. The above proposition indicates that such a classification would be rather involved.

It would be interesting to classify the total spaces of $S^{3}$-bundles over $S^{4}$ with nonzero Euler class up to tangential homotopy equivalence. Indeed, by Masur's theorem such a classification should lead to an analog of Proposition 3 , with $S^{7}$ replaced by any $S^{3}$-bundle $S$ over $S^{4}$ with nonzero Euler class. It is worth mentioning that nonvanishing of the Euler class implies that the homotopy type of $S$ contains at most finitely many nondiffeomorphic $S^{3}$-bundles over $S^{4}$ [Tam58] (cf. [CE00]).

It is easy to construct manifolds admitting two nonnegatively curved metrics with pairwise nonhomeomorphic souls. Here we describe two such situations where souls are lens spaces, or simply-connected homogeneous manifolds.

Example 4. It is well known [Coh73, pp. 96, 100] that the lens spaces $L(7,1)$ and $L(7,2)$ are homotopy equivalent, but not simply-homotopy equivalent (hence nonhomeomorphic). Orientable 3 -manifolds are parallelizable, so any homotopy equivalence of $L(7,1), L(7,2)$ is tangential, and therefore [Sie69, Theorem 2.3] we get a diffeomorphism of manifolds $L(7,1) \times \mathbb{R}^{4}$ and $L(7,2) \times \mathbb{R}^{4}$ admitting obvious product metrics of sec $\geq 0$ with souls $L(7,1) \times\{0\}, L(7,2) \times\{0\}$. Since every homotopy type contains at most finitely many nonhomeomorphic lens spaces, this procedure yields only finitely many pairwise nonhomeomorphic souls on a given manifold.

Proposition 5. If $i$ is a positive integer divisible by 24, then there is a compact homogeneous space $G / H$ homotopy equivalent but not homeomorphic to $S^{4 i-1} \times S^{4}$, such that for $n>4 i+3$, the manifold $G / H \times \mathbb{R}^{n}$ carries two complete nonnegatively curved metrics with souls diffeomorphic to $G / H$ and $S^{4 i-1} \times S^{4}$.

The above-mentioned result of Siebenmann [Sie69, Theorem 2.2] states that if $\xi$ is a rank $>2$ vector bundle over a compact manifold, and the total space $E(\xi)$ of $\xi$ contains a smooth compact submanifold $S$ such that the inclusion $S \rightarrow E(\xi)$ is a homotopy equivalence, then $E(\xi)$ has a vector bundle structure with zero section $S$.

Then it easily follows [Sie69, Theorem 2.3] that if $t$ is tangential homotopy equivalence of $n$-manifolds, each being the total space of a vector bundle over a compact smooth manifold of dimension less than the rank of the bundle, then $t$ is homotopic to a diffeomorphism. The upshot is that the total space of vector bundle of sufficiently large rank often has many other vector bundle structures, perhaps with nonhomeomorphic base spaces, and this fact plays a crucial role in this note.

Proof of Theorem 0 . In what follows we denote the total space of a vector bundle $\zeta$ by $E(\zeta)$, and the associated sphere bundle by $S(\zeta)$. Principal $S O(3)$-bundles 
over $S^{4}$ are in one-to-one correspondence with $\pi_{4}(B S O(3)) \cong \mathbb{Z}$. Let $P_{k}$ be the principal $S O(3)$-bundle over $S^{4}$ corresponding to $k \in \pi_{4}(B S O(3))$. Let $\xi_{k}^{n}$ be the rank $n$ vector bundle over $S^{4}$ associated with $P_{k}$ via the standard inclusion $S O(3) \rightarrow S O(n)$.

Let $P_{k, l}$ be the principal $S O(3) \times S O(3)$-bundle over $S^{4}$ which is the pullback of $P_{k} \times P_{l}$ via the diagonal map $\triangle: S^{4} \rightarrow S^{4} \times S^{4}$. According to GZ00, $P_{k, l}$ admits an $S O(3) \times S O(3)$-invariant metric with sec $\geq 0$. Consider the $S^{m-1} \times \mathbb{R}^{n}$-bundle

$$
\triangle^{\#}\left(S\left(\xi_{k}^{m}\right) \times \xi_{l}^{n}\right)=S^{m-1} \times_{S O(3) \times 1} P_{k, l} \times_{1 \times S O(3)} \mathbb{R}^{n}
$$

associated with $P_{k, l}$. The total space of $\triangle^{\#}\left(S\left(\xi_{k}^{m}\right) \times \xi_{l}^{n}\right)$ is also the total space of a rank $n$ vector bundle over $S\left(\xi_{k}^{m}\right)$ which we denote by $\eta_{k, l}^{m, n}$. Note that $\eta_{k, l}^{m, n}$ is the pullback of $\xi_{l}^{n}$ via the projection $q_{k}^{m}: S\left(\xi_{k}^{m}\right) \rightarrow S^{4}$. By O'Neill's formula for submersions, $E\left(\eta_{k, l}^{m, n}\right)$ carries a complete metric with sec $\geq 0$ with zero section being a soul.

First, note that $S\left(\xi_{k}^{m}\right)$ is fiber homotopy equivalent to $S\left(\xi_{i}^{m}\right)$ if $k \equiv i(\bmod 12)$. Indeed, $S^{m-1}$-fibrations over $S^{4}$ are classified, up to fiber homotopy equivalence, by $\pi_{3}\left(S G_{m}\right)$ where $S G_{m}$ is the space of orientation-preserving self-homotopy equivalences of $S^{m-1}$. The fibrations $S\left(\xi_{k}^{m}\right)$ are classified by the image of

$$
\phi: \pi_{3}(S O(3)) \rightarrow \pi_{3}(S O(m)) \rightarrow \pi_{3}\left(S G_{m}\right) .
$$

Since $m \geq 4, \phi$ factors as $\pi_{3}(S O(3)) \rightarrow \pi_{3}\left(S F_{3}\right) \rightarrow \pi_{3}\left(S G_{4}\right) \rightarrow \pi_{3}\left(S G_{m}\right)$ where $S F_{3}$ is the space of base-point-preserving elements of $S G_{4}$. It is well known that $S F_{3}$ is the identity component of the loop space $\Omega^{3} S^{3}$ [MM79, Chapter 3], hence $\pi_{3}\left(S F_{3}\right) \cong \pi_{6}\left(S^{3}\right)$. Thus, $\phi$ factors through the $J$-homomorphism $\mathbb{Z} \cong \pi_{3}(S O(3)) \rightarrow$ $\pi_{6}\left(S^{3}\right) \cong \mathbb{Z}_{12}$, and the result follows.

Second, show that $S\left(\xi_{k}^{m}\right)$ is homeomorphic to $S\left(\xi_{i}^{m}\right)$ iff $k= \pm l$ iff $S\left(\xi_{k}^{m}\right)$ is diffeomorphic to $S\left(\xi_{l}^{m}\right)$. Indeed, the tangent bundle of $S\left(\xi_{k}^{m}\right)$ is stably isomorphic to the $q_{k}^{m}$-pullback of $\xi_{k}^{m}$, because $S\left(\xi_{k}^{m}\right)$ is a two-sided hypersurface in $E\left(\xi_{k}^{m}\right)$. Since $\xi_{k}^{m}$ is an $S O(3)$-bundle, the first Pontrjagin class of $\xi_{k}^{m}$ is the $\pm 4 k$-multiple of a generator of $H^{4}\left(S^{4}, \mathbb{Z}\right)$ (cf. Mil56]). Also $S\left(\xi_{k}^{m}\right)$ has a section so that $q_{k}^{m}$ induces an isomorphism on the 4 th cohomology, hence $p_{1}\left(T S\left(\xi_{k}^{m}\right)\right)$ is the $\pm 4 k$-multiple of a generator. By the topological invariance of rational Pontrjagin classes, $S\left(\xi_{k}^{m}\right)$ is not homeomorphic to $S\left(\xi_{i}^{m}\right)$ unless $k= \pm i$. Finally, $P_{k}$ is the pullback of $P_{-k}$ via an orientation-reversing self-diffeomorphism of $S^{4}$, so $S\left(\xi_{k}^{m}\right)$ and $S\left(\xi_{-k}^{m}\right)$ are diffeomorphic.

Third, note that $E\left(\eta_{k, l}^{m, n}\right)$ and $E\left(\eta_{i, j}^{m, n}\right)$ are tangentially homotopy equivalent provided $k \equiv i(\bmod 12)$, and $k+l=i+j$. Indeed, fix $k, l, i, j$ with these properties. The tangent bundle to $E\left(\eta_{k, l}^{m, n}\right)$ is determined by its restriction to the zero section. This restriction is the $q_{k}^{m}$-pullback of $\xi_{k}^{m} \oplus \xi_{l}^{n}$ for any $k, l$. The images of $\xi_{k}^{m}, \xi_{l}^{n}$ under the homomorphism

$$
\pi_{4}(B S O(3)) \rightarrow \pi_{4}(B S O)
$$

add up to $k+l$, and the addition in $\pi_{4}(B S O)$ is given by the Whitney sum $\oplus$. Thus $k+l=i+j$ implies that $\xi_{k}^{m} \oplus \xi_{l}^{n}$ and $\xi_{i}^{m} \oplus \xi_{j}^{n}$ are stably isomorphic. Since $k \equiv i(\bmod 12)$ there is a fiber homotopy equivalence $g: S\left(\xi_{k}^{m}\right) \rightarrow S\left(\xi_{i}^{m}\right)$, so that $g \circ q_{i}^{m}$ is homotopic to $q_{k}^{m}$. Therefore, $g$ induces a tangential homotopy equivalence $t: E\left(\eta_{k, l}^{m, n}\right) \rightarrow E\left(\eta_{i, j}^{m, n}\right)$, which is the composition of the projection of $\eta_{k, l}^{m, n}$, followed by $g$, and then by the zero section of $\eta_{i, j}^{m, n}$. 
Next we show that $E\left(\eta_{k, l}^{m, n}\right)$ and $E\left(\eta_{i, j}^{m, n}\right)$ are diffeomorphic if $k \equiv i(\bmod 12)$, and $k+l=i+j$. By Haefliger's embedding theorem [Hae61, the restriction of $t$ to the zero section of $\eta_{k, l}^{m, n}$ is homotopic to a smooth embedding $f$ because $2 n \geq m+6$, which means we are in metastable range. Since $n \geq 3$, [Sie69, Theorem 2.2] implies that $E\left(\eta_{i, j}^{m, n}\right)$ has a vector bundle structure with zero section $f$. Since $t$ is tangential, $\eta_{k, l}^{m, n}$ is stably isomorphic to $\nu_{f}$ which is the normal bundle to $f$.

In fact, our assumptions on $n, m$ imply that $\eta_{k, l}^{m, n}$ and $\nu_{f}$ are isomorphic. Indeed, if $m>4, n>m+3=\operatorname{dim}\left(S\left(\xi_{k}^{m}\right)\right)$, then we are in stable range, hence $\eta_{k, l}^{m, n} \cong \nu_{f}$. If $m=4, n>4$, we apply obstruction theory comparing $\nu_{f}, \eta_{k, l}^{m, n}$, which are thought of as classifying maps from $S\left(\xi_{k}^{m}\right)$ to $B S O(n)$. Since $S\left(\xi_{k}^{m}\right)$ has a section, it can be obtained by attaching a 7-cell to $S^{3} \vee S^{4}$. The bundles $\nu_{f}, \eta_{k, l}^{m, n}$ are isomorphic on the 6-skeleton $S^{3} \vee S^{4}$, because they are stably isomorphic and $n>$ $4=\operatorname{dim}\left(S^{3} \vee S^{4}\right)$. Comparing $\nu_{f}, \eta_{k, l}^{m, n}$ on the 7-cell, we get a map $S^{7} \rightarrow B S O(n)$ which is nullhomotopic since $\pi_{7}(B S O(n))=0$ if $n>4$ [Mim95. page 970]). Thus, $\nu_{f}, \eta_{k, l}^{m, n}$ are isomorphic, and hence $E\left(\eta_{k, l}^{m, n}\right)$ and $E\left(\eta_{i, j}^{m, n}\right)$ are diffeomorphic.

To summarize, each manifold $E\left(\eta_{k, l}^{m, n}\right)$ has infinitely many vector bundle structures $\eta_{i, j}^{m, n}$ with base manifolds $S\left(\xi_{i}^{m}\right)$ for any $i, j$ satisfying $k \equiv i(\bmod 12)$, and $k+l=i+j$, and the proof is complete.

Proof of Proposition [3. Let $\Sigma$ be a homotopy 7-sphere of sec $\geq 0$ [GM74, GZ00]. The product metric on $\Sigma \times \mathbb{R}^{n}$ has sec $\geq 0$ with a soul $\Sigma \times\{0\}$. By [Hae61] any homotopy equivalence $S^{7} \rightarrow \Sigma \times \mathbb{R}^{n}$ is homotopic to a smooth embedding if $n \geq 5$, so $\Sigma \times \mathbb{R}^{n}$ gets a structure of a vector bundle over $S^{7}$ which is necessarily trivial since $\pi_{7}(B S O(n))=0$ for $n \geq 5$. Thus, $\Sigma \times \mathbb{R}^{5}$ is diffeomorphic to $S^{7} \times \mathbb{R}^{5}$, as promised.

Proof of Proposition [5, Kamerich [Kam77, page 116] (cf. [Oni94, page 275]) showed that if $i$ is a positive integer divisible by 24, then there is a compact homogeneous space $G / H$ homotopy equivalent but not homeomorphic to $S^{4 i-1} \times S^{4}$. Here $G=$ $S p(i) \times S p(2)$ and $H=S p(i-1) \times S p(1) \times S p(1)$, where $S p(i-1)$ is embedded in $S p(i)$ is the standard way, the first $S p(1)$ is embedded into $S p(i) \times S p(2)$ diagonally so that a quaternion goes to the last diagonal entry of the matrix in $S p(i)$, and also to the first diagonal entry in $S p(2)$, while the second $S p(1)$ goes into the last diagonal entry of $S p(2)$.

Since $n>4 i+3$, the homotopy equivalence $S^{4 i-1} \times S^{4} \rightarrow G / H \times \mathbb{R}^{n}$ is homotopic to a smooth embedding $f$. By [Sie69, Theorem 2.2], $G / H \times \mathbb{R}^{n}$ admits an $\mathbb{R}^{n}$-bundle structure with zero section $f$.

Note that any $\mathbb{R}^{n}$-bundle $\xi$ over $S^{4 i-1} \times S^{4}$ is the pullback of a $\mathbb{R}^{n}$-bundle over $S^{4}$ via the projection $S^{4 i-1} \times S^{4} \rightarrow S^{4}$. This is proved by obstruction theory for maps $S^{4 i-1} \times S^{4} \rightarrow B S O$ by comparing $\xi$ with the bundle $\xi_{4}$ obtained by pullbacking $\xi$ to $S^{4}$ via an inclusion $S^{4} \rightarrow S^{4 i-1} \times S^{4}$, and then pullbacking it back to $S^{4 i-1} \times S^{4}$ via the projection $S^{4 i-1} \times S^{4} \rightarrow S^{4}$. Since $\pi_{4 i-1}(B S O)=0, \xi$ and $\xi_{4}$ agree on $S^{4 i-1} \vee S^{4}$, and they are homotopic on the top $4 i+3$-cell as $\pi_{4 i+3}(B S O)=0$.

Since each vector bundle over $S^{4}$ carries sec $\geq 0$ with zero section being a soul GZ00, so does the product of the bundle and $S^{4 i-1}$. Thus $G / H \times \mathbb{R}^{n}$ gets a metric with sec $\geq 0$ and soul $S^{4 i-1} \times S^{4}$. On the other hand, $G / H \times \mathbb{R}^{n}$ has the product metric with soul $G / H$. 


\section{ACKNOWLEDGEMENTS}

The results of this paper were obtained during a geometry meeting in Oberwolfach in June 2001, and the author is grateful to the meeting organizers for excellent working conditions and hospitality. Special thanks are due to Wolfgang Ziller for insightful discussions and encouragement, and to McKenzie Wang for helpful communications about Kamerich's thesis.

\section{REFERENCES}

[CE00] D. Crowley and C. M. Escher, A classification of $S^{3}$-bundles over $S^{4}$, to appear in Differential Geom. Appl.

[CG72] J. Cheeger and D. Gromoll, On the structure of complete manifolds of nonnegative curvature, Ann. of Math. 96 (1972), 413-443. MR 46:8121

[Coh73] M. M. Cohen, A course in simple-homotopy theory, Springer-Verlag, 1973. MR 50:14762

[GM74] D. Gromoll and W. Meyer, An exotic sphere with nonnegative sectional curvature, Ann. of Math. 100 (1974), 401-406. MR 51:11347

[GT01] D. Gromoll and K. Tapp, Nonnegatively curved metrics on $S^{2} \times \mathbb{R}^{2}$, to appear in Geom. Dedicata.

[GZ00] K. Grove and W. Ziller, Curvature and symmetry of Milnor spheres, Ann. Math. 151 (2000), 1-36. MR 2000i:53047

[Hae61] A. Haefliger, Plongements différentiables de variétés dans variétés, Comment. Math. Helv. 36 (1961), 47-82.

[Kam77] B. N. P. Kamerich, Transitive transformation groups of products of two spheres, Ph.D. thesis, Catholic University of Nijmegen, 1977.

[Mil56] J. Milnor, On manifolds homeomorphic to the 7-sphere, Ann. of Math. 64 (1956), 399405. MR 18:498d

[Mim95] M. Mimura, Homotopy theory of Lie groups, Handbook of algebraic topology, NorthHolland, 1995, pp. 951-991. MR 97c:57038

[MM79] I. Madsen and R. J. Milgram, The classifying spaces for surgery and cobordism of manifolds, Princeton University Press, 1979. MR 81b:57014

[Oni94] A. L. Onishchik, Topology of transitive transformation groups, Johann Ambrosius Barth Verlag GmbH, 1994. MR 95e:57058

[Sha79] V. A. Sharafutdinov, Convex sets in a manifold of nonnegative curvature, Math. Notes 26 (1979), no. no. 1-2, 556-560. MR 81d:53039

[Sie69] L. Siebenmann, On detecting open collars, Trans. Amer. Math. Soc. 142 (1969), 201227. MR 39:7605

[Tam58] I. Tamura, Homeomorphy classification of total spaces of sphere bundles over spheres, J. Math. Soc. Japan 10 (1958), 29-43. MR 20:2717

Department of Mathematics, 253-37, California Institute of Technology, Pasadena, California 91125

E-mail address: ibeleg@its.caltech.edu 